\title{
Beyond the fold approximation: the extended-source effect in two-point-mass microlensing
}

\section{Ondřej Pejcha* and David Heyrovský}

Institute of Theoretical Physics, Charles University in Prague, Czech Republic

E-mails: pejco3amekolej.mff.cuni.cz, heyrovsky@utf.mff.cuni.cz

\begin{abstract}
We have implemented an efficient method for computing the amplification of an extended source with an arbitrary surface brightness profile by a two-point-mass lens. Using our code we study the sensitivity to an extended source for various binary lens configurations and find previously unknown areas of enhanced sensitivity. In addition we investigate the influence of limb darkening on binary microlensing (chromaticity). We provide analytical approximations for both the sensitivity to an extended source and chromaticity. Finally, we compare the linear fold approximation of caustic crossing to the exact light curve for a binary event that was previously used to infer limb-darkening coefficients of the source. We demonstrate that deviations from the linear fold are observationally significant.
\end{abstract}

The Manchester Microlensing Conference: The 12th International Conference and ANGLES Microlensing Workshop

January 21-25 2008

Manchester, $U K$

* Speaker. 


\section{Introduction}

Although gravitational microlensing by two point masses comprises a minority of the observed events, the amount of knowledge that can be obtained through their study is significant. Finding extrasolar planets with microlensing has attracted particular attention. During the brief planetary perturbations to the light curve the source cannot be regarded as point-like and a correct limbdarkening model is necessary to properly interpret the light curves. In other words, changes of gradient of amplification in binary microlensing spatially resolve the source. That enables testing of the stellar atmosphere models by actual reconstruction of the surface brightness profile and mapping of the spectral line formation by observations of spectral changes. In our work we map the extended-source effect in the two-point-mass lens geometry and investigate the chromaticity. We also check if a linear fold approximation produces reasonable results.

The lens equation of a two-point-mass lens is

$$
\mathbf{y}=\mathbf{x}-\mu_{A} \frac{\mathbf{x}-\mathbf{x}_{A}}{\left|\mathbf{x}-\mathbf{x}_{A}\right|^{2}}-\mu_{B} \frac{\mathbf{x}-\mathbf{x}_{B}}{\left|\mathbf{x}-\mathbf{x}_{B}\right|^{2}}
$$

where $\mu_{A}$ and $\mu_{B}$ are the masses of the lens components relative to their total mass $M\left(\mu_{A}+\mu_{B}=1\right)$, and $\mathbf{x}_{A}$ and $\mathbf{x}_{B}$ denote the locations of the components. Angular positions $\mathbf{y}, \mathbf{x}, \mathbf{x}_{A}$ and $\mathbf{x}_{B}$ are expressed in units of the angular Einstein radius of the compound lens. Amplification $A_{*}$ of an extended source centered at $\mathbf{y}_{\mathrm{c}}$ is given by the cross-correlation of the point-source amplification $A_{0}$ and the surface-brightness distribution of the source $B$ normalized by the unamplified flux

$$
A_{*}\left(\mathbf{y}_{\mathrm{c}}\right)=\frac{\int_{\Sigma_{\mathrm{S}}} A_{0}\left(\mathbf{y}_{\mathrm{c}}+\mathbf{y}^{\prime}\right) B\left(\mathbf{y}^{\prime}\right) \mathrm{d}^{2} \mathbf{y}^{\prime}}{\int_{\Sigma_{\mathrm{S}}} B\left(\mathbf{y}^{\prime}\right) \mathrm{d}^{2} \mathbf{y}^{\prime}}=\frac{\int_{\Sigma_{\mathrm{I}}} B\left(\mathbf{y}[\mathbf{x}]-\mathbf{y}_{\mathrm{c}}\right) \mathrm{d}^{2} \mathbf{x}}{\int_{\Sigma_{\mathrm{S}}} B\left(\mathbf{y}^{\prime}\right) \mathrm{d}^{2} \mathbf{y}^{\prime}} .
$$

Here $\mathbf{y}^{\prime}$ is measured from the source center and the integration is performed over the area of the source $\Sigma_{\mathrm{S}}$. To convert the numerator to the second expression we set $\mathbf{y}^{\prime}=\mathbf{y}[\mathbf{x}]-\mathbf{y}_{\mathrm{c}}$ and transform the integral to image coordinates, integrating over the area of the images $\Sigma_{\mathrm{I}}$. In this way $A_{0}$ cancels out with the Jacobian of the transformation. The relation between the position of the source at $\mathbf{y}[\mathbf{x}]$ and of the image at $\mathbf{x}$ is given by the lens equation (1.1).

Computation of $A_{*}$ poses significant numerical difficulties, especially for arbitrary $B$. Here we use the method suggested by Vermaak (2000) and described in detail in Pejcha \& Heyrovský (2007, hereafter PH07). The method combines direct inversion of the lens equation and ray shooting. We find image positions of the center of the source $\mathbf{y}_{\mathrm{c}}$ through inversion of the lens equation (1.1) and then start a recursive flood-fill ray-shooting algorithm to go through the images. Two extra images appear when an edge of the source enters the caustic. These images may be detached from the other three and would be missed. To take them into account, we compute the intersection of the circumference of the source with the caustic curve and restart the algorithm from a point just interior of such an intersection.

We model the source by a circular disk with radius $\rho_{*}$ in Einstein angle units and we use radially symmetric limb darkening $B\left(\mathbf{y}^{\prime}\right)=I(r), r=\left|\mathbf{y}^{\prime}\right| / \rho_{*}$. We assume that $I(r)$ is given by a linear combination of two orthonormal functions

$$
I(r)=c_{1} f_{1}(r)+c_{2} f_{2}(r)=c_{1}\left[f_{1}(r)+\kappa f_{2}(r)\right]
$$



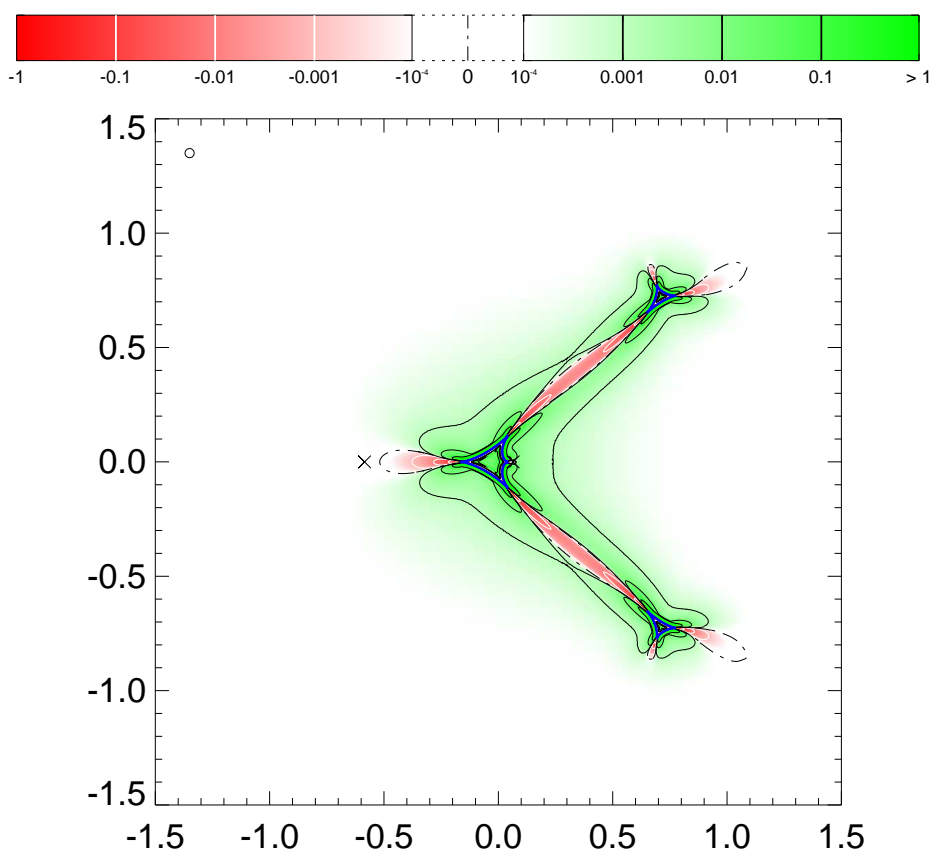

Figure 1: Sensitivity to an extended source $\delta_{\mathrm{ex}}$ as a function of the source-center position $\mathbf{y}_{\mathrm{c}}$ for a lens with $q=1 / 9$ and $d=0.65$. Sensitivity is both plotted with contours and marked in color as shown in the color bar above. Dot-dashed lines denote places with zero effect $\delta_{\mathrm{ex}}=0$. Caustic curves are shown with blue lines and the positions of the point masses are marked with crosses. The circle in the top left illustrates the size of the source.

that were obtained by principal component analysis (PCA) of Kurucz's ATLAS model atmospheres (PH07). Plausible $\kappa$ values span the interval from $\kappa_{\mathrm{pk}}=-0.1620$ for the peakiest profile to $\kappa_{\mathrm{fl}}=$ 0.0902, that describes the flattest allowed limb darkening.

\section{Sensitivity to an extended source}

\subsection{Areas of sensitivity}

To map the sensitivity to an extended source we use only the first principal function ${ }^{1}$ for limb darkening, hence $\kappa=0$. For a source at $\mathbf{y}_{\mathrm{c}}$ we define the sensitivity to an extended source as a relative amplification difference of $A_{*}$ over the point-source amplification

$$
\delta_{\mathrm{ex}}\left(\mathbf{y}_{\mathrm{c}}\right) \equiv \frac{A_{*}\left(\mathbf{y}_{\mathrm{c}}\right)-A_{0}\left(\mathbf{y}_{\mathrm{c}}\right)}{A_{0}\left(\mathbf{y}_{\mathrm{c}}\right)}
$$

A contour plot of $\delta_{\mathrm{ex}}$ for a binary lens with mass ratio $q=1 / 9$ and separation between the two point masses $d=0.65$ in Einstein angle units is given in Figure 1. The source radius is $\rho_{*}=0.02$ which corresponds to a typical giant source. We see that most of the area is green where $\delta_{\mathrm{ex}}>0$ and hence $A_{*}>A_{0}$. In these areas the drop of $A_{0}$ from the caustic is convex and some parts of the source are

\footnotetext{
${ }^{1}$ weighted average shape of the set of model atmospheres
} 


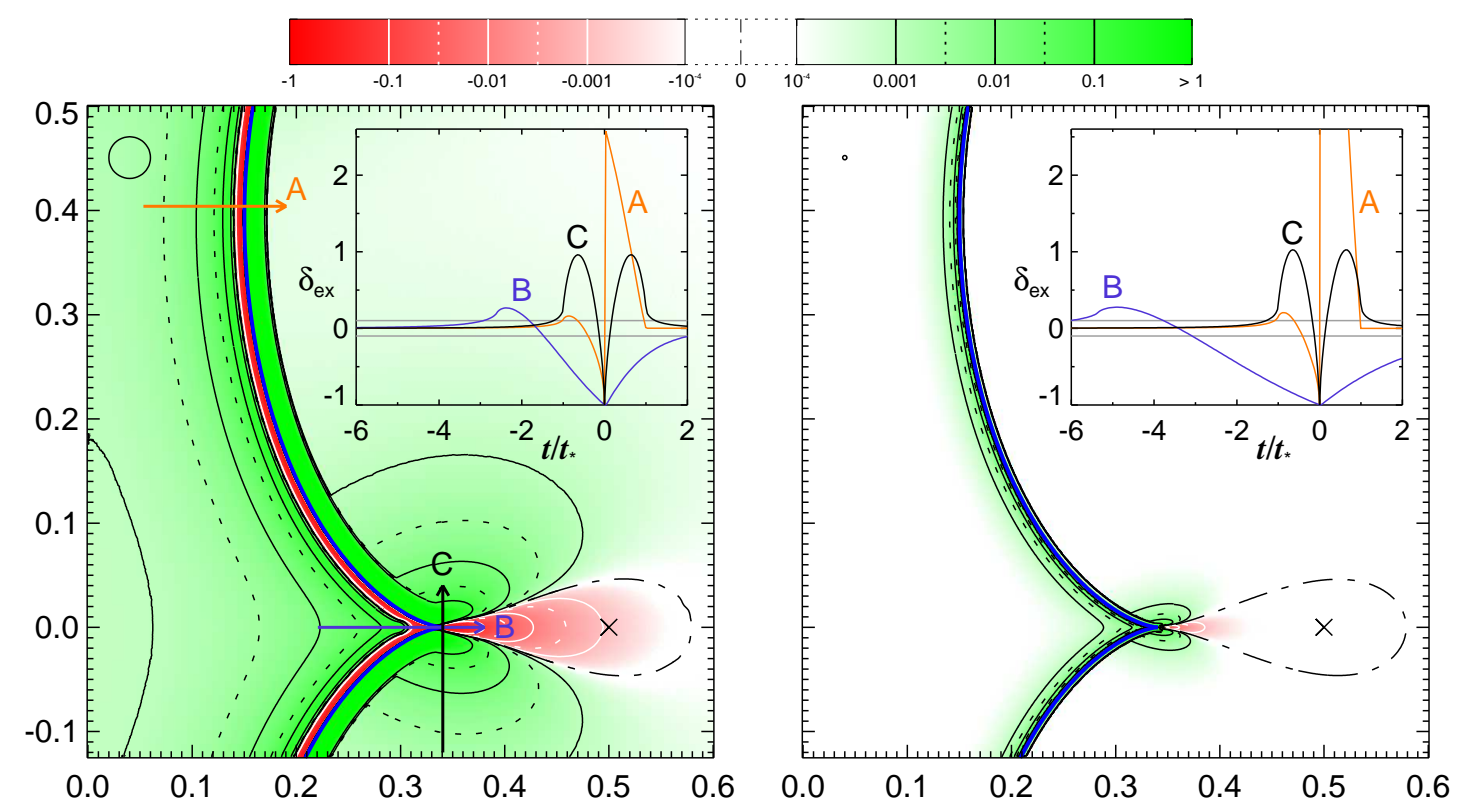

Figure 2: Sensitivity to an extended source for a lens with $q=d=1$ and sources with $\rho_{*}=0.02$ (left panel) and $\rho_{*}=0.002$ (right panel). Symbols have the same meaning as in Figure 1 except that four extra contours were added. Cuts through the $\delta_{\mathrm{ex}}$ surface are marked with arrows and are shown also in the inset plots. The lengths of the cuts are the same in units of source radius in both panels. The cuts are parameterized by $t / t_{*}$ where $t_{*}$ is the time for a source to cross its radius; $t=0$ corresponds to a source positioned on the fold or on the cusp, respectively.

always closer to the caustic than the center. The limited red areas where $A_{*}<A_{0}$ are seen along the outer axes of the cusps. Here the concave perpendicular drop of $A_{0}$ is initially stronger than the convex drop along the axis. Neglecting this perpendicular drop can lead to incorrect results on the amplification of an extended source along the axis of the cusp (for more details see PH07).

The most interesting features are the red areas that connect facing cusps of the caustic components. The sensitivity extends many source radii from the cusp and the magnitude of the effect can reach well over $1 \%$. These regions are observed not only in the three-part caustics of smallseparation binaries but also on the lens axis for wide binaries with two-part caustics. The strength and size of the regions depend on $q$ and $d$. Keeping $d$ fixed and lowering $q$, the caustic components become smaller and more separated and the negative regions get more diffuse and perpendicularly thinner. For fixed $q$ there exist values of $d$ where the negative region splits into two opposite lobes. Sensitive areas between the facing cusps can significantly increase the probability of observing the extended-source effect in comparison with pure caustic crossing. For this particular lens configuration the mean projected width of the $\left|\delta_{\mathrm{ex}}\right|=1 \%$ contour is 2.08 times higher than the width of the caustic curves increased by the source size. For the $\left|\delta_{\text {ex }}\right|=10 \%$ effect the width is still about $30 \%$ higher. The projected widths are defined in a similar way as in Mao \& Paczyński (1991).

To investigate the behavior of $A_{*}$ in the vicinity of cusps more thoroughly, we plot in Figure 2 a detail of a lens with $q=d=1$. We see that there is a band of negative $\delta_{\text {ex }}$ just inside the caustic curves. As the source approaches the caustic from inside, the point-source amplification gradually 
rises to the divergence right on the caustic, where the lowest possible value of $\delta_{\mathrm{ex}}=-1$ is achieved. To study the scaling of the structures with source radius we take three cuts through the $\delta_{\text {ex }}$ surface that go perpendicularly to the fold (A), along the cusp axis (B) and perpendicularly to it (C). The lengths of the cuts are constant in units of source radii. We see that cut $\mathrm{C}$ scales almost perfectly with source size and the two positive peaks occur approximately $0.65 \rho_{*}$ off the cusp. Cut A scales almost perfectly with source radius, too, albeit the height of the second maximum changes as $\rho_{*}^{-1 / 2}$. However, cut B going along the axis of the cusp is significantly wider for the smaller source. Inside the cusp the smaller source touches the convexly shaped caustic relatively sooner that the larger source. Outside the cusp the contour of $-1 \%$ effect is located $4.4 \rho_{*}$ from the cusp for the larger source but at $11 \rho_{*}$ for the smaller source.

\subsection{Analytical estimates}

To explain the results from the preceding section theoretically, we follow PH07, and under assumption that the source is small and is positioned off the caustic, we expand the integrand of the first expression of equation (1.2) at the source center $\mathbf{y}_{\mathrm{c}}$. We get the following expression for the extended-source amplification

$$
A_{*}\left(\mathbf{y}_{\mathrm{c}}\right)=A_{0}\left(\mathbf{y}_{\mathrm{c}}\right)+\frac{1}{4} \Delta A_{0}\left(\mathbf{y}_{\mathrm{c}}\right) \rho_{*}^{2} \frac{\int_{0}^{1} I(r) r^{3} \mathrm{~d} r}{\int_{0}^{1} I(r) r \mathrm{~d} r}+\frac{1}{64} \Delta^{2} A_{0}\left(\mathbf{y}_{\mathrm{c}}\right) \rho_{*}^{4} \frac{\int_{0}^{1} I(r) r^{5} \mathrm{~d} r}{\int_{0}^{1} I(r) r \mathrm{~d} r}+O\left(\rho_{*}^{6}\right),
$$

where $\Delta$ is the Laplacian and $\Delta^{2}=\Delta \Delta$ is the biharmonic operator. This leads to an approximate expression for the sensitivity to an extended source

$$
\delta_{\mathrm{ex}}\left(\mathbf{y}_{\mathrm{c}}\right) \approx \frac{\Delta A_{0}\left(\mathbf{y}_{\mathrm{c}}\right)}{4 A_{0}\left(\mathbf{y}_{\mathrm{c}}\right)} \rho_{*}^{2} \frac{\int_{0}^{1} I(r) r^{3} \mathrm{~d} r}{\int_{0}^{1} I(r) r \mathrm{~d} r} .
$$

We see that the dependence on limb darkening factors out to a constant and that the shape of the contours depends only on the lens geometry. The Laplacian of $A_{0}$ in equation (2.3) advises us that when investigating the extended-source amplification along a particular line we cannot neglect the behaviour of $A_{0}$ in the perpendicular direction.

Here we focus on the behavior of $\delta_{\mathrm{ex}}$ along the outer axis of a generic cusp. We use the pointsource amplification formulae of Schneider \& Weiß (1992) and Zakharov $(1995,1999)$ that are summarized in PH07. For a source positioned on the outer axis of cusp at a distance $y_{\|}$we arrive at the following expression

$$
\frac{\Delta A_{0}}{A_{0}}=\frac{8 K}{9 y_{\|}^{3}}+\frac{2}{y_{\|}^{2}},
$$

where $K$ is a parameter that controls the orientation and narrowness of a cusp. The first term proportional to $y_{\|}^{-3}$ comes from the derivatives in the direction perpendicular to $y_{\|}$and has paramount importance in the vicinity of the cusp. Note that the first term is negative, since $K / y_{\|}<0$ along the outer cusp axis. Neglecting the second term and plugging into equation (2.3) we arrive at an expression for the extent of a contour of value $\delta_{\mathrm{ex}}$ along the outer axis of the cusp

$$
\frac{\mathrm{y}_{\|}}{\rho_{*}} \approx\left[\frac{2 K}{9 \rho_{*} \delta_{\mathrm{ex}}} \frac{\int_{0}^{1} I(r) r^{3} \mathrm{~d} r}{\int_{0}^{1} I(r) r \mathrm{~d} r}\right]^{1 / 3} .
$$

When expressed in units of source radii the contour extent scales as $\rho_{*}^{-1 / 3}$ which confirms the observation made in the preceding section. 


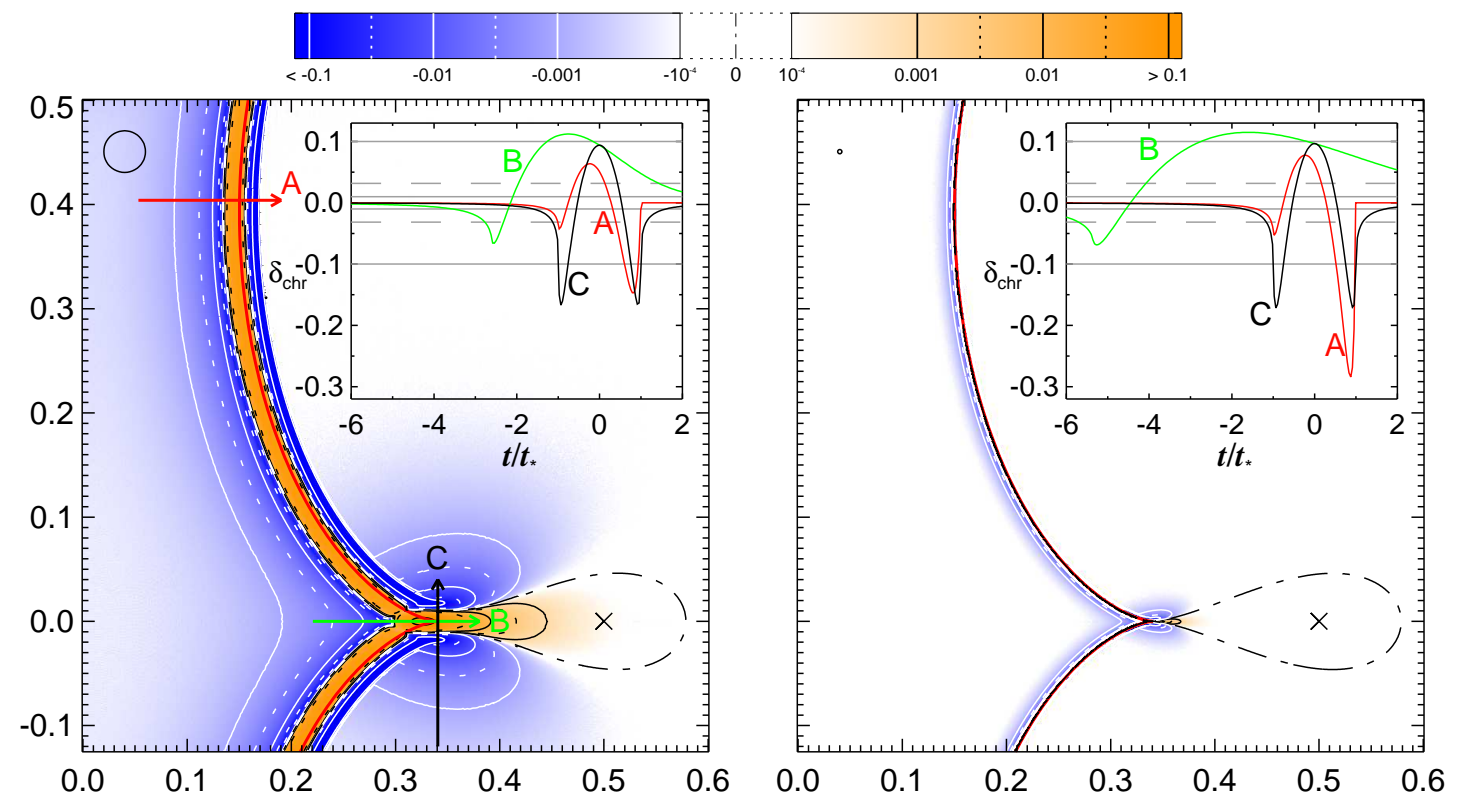

Figure 3: Chromaticity $\delta_{\mathrm{chr}}$ as a function of source position $\mathbf{y}_{\mathrm{c}}$ for a lens with $d=q=1$ and source radii $\rho_{*}=0.02$ (left panel) and $\rho_{*}=0.002$ (right panel). Contour values and the coding of colors is shown in the color bar above. Cuts through the chromaticity surface are at the same positions as in Figure 2. Meaning of other symbols is similar to those of Figure 2.

\section{Chromaticity}

Observing a microlensing event in different spectral passbands can lead to color variations in the light curve. We define chromaticity $\delta_{\text {chr }}$ as the relative amplification difference of two sources with extreme limb-darkening profiles

$$
\delta_{\mathrm{chr}}\left(\mathbf{y}_{\mathrm{c}}\right) \equiv \frac{A_{*}\left(\mathbf{y}_{\mathrm{c}}, \kappa_{\mathrm{pk}}\right)-A_{*}\left(\mathbf{y}_{\mathrm{c}}, \kappa_{\mathrm{ff}}\right)}{A_{*}\left(\mathbf{y}_{\mathrm{c}}, 0\right)} .
$$

In a manner similar to and under the same assumptions as in section 2.2 we found (PH07) that chromaticity defined in this way can be approximated away from the caustic by $\delta_{\text {ex }}$ multiplied by a constant that depends only on the assumed form of limb darkening

$$
\delta_{\mathrm{chr}}\left(\mathbf{y}_{\mathrm{c}}\right) \approx h\left(\kappa_{\mathrm{pk}}, \kappa_{\mathrm{fl}}\right)\left[\frac{\int_{0}^{1} f_{2}(r) r^{3} \mathrm{~d} r \int_{0}^{1} f_{1}(r) r \mathrm{~d} r}{\int_{0}^{1} f_{2}(r) r \mathrm{~d} r \int_{0}^{1} f_{1}(r) r^{3} \mathrm{~d} r}-1\right] \delta_{\mathrm{ex}}\left(\mathbf{y}_{\mathrm{c}}\right) .
$$

For our particular choice of limb darkening we have $\delta_{\text {chr }} \approx-0.197 \delta_{\mathrm{ex}}$. The discussion of the shapes of $\delta_{\text {chr }}$ contours is similar to section 2.1 keeping in mind that the peakiest profile is a better proxy to the point source than the flattest profile.

The behavior of chromaticity close to the caustic is different from the sensitivity to an extended source. In Figure 3 we plot chromaticity for the same lens detail as in Figure 2. Because chromaticity is constructed from extended-source amplifications that don't diverge on the caustic, the behavior is smooth. Regions of maximum positive chromaticity are located just inside the cusps (where the central parts of the source receive highest amplification) while the maximum negative 

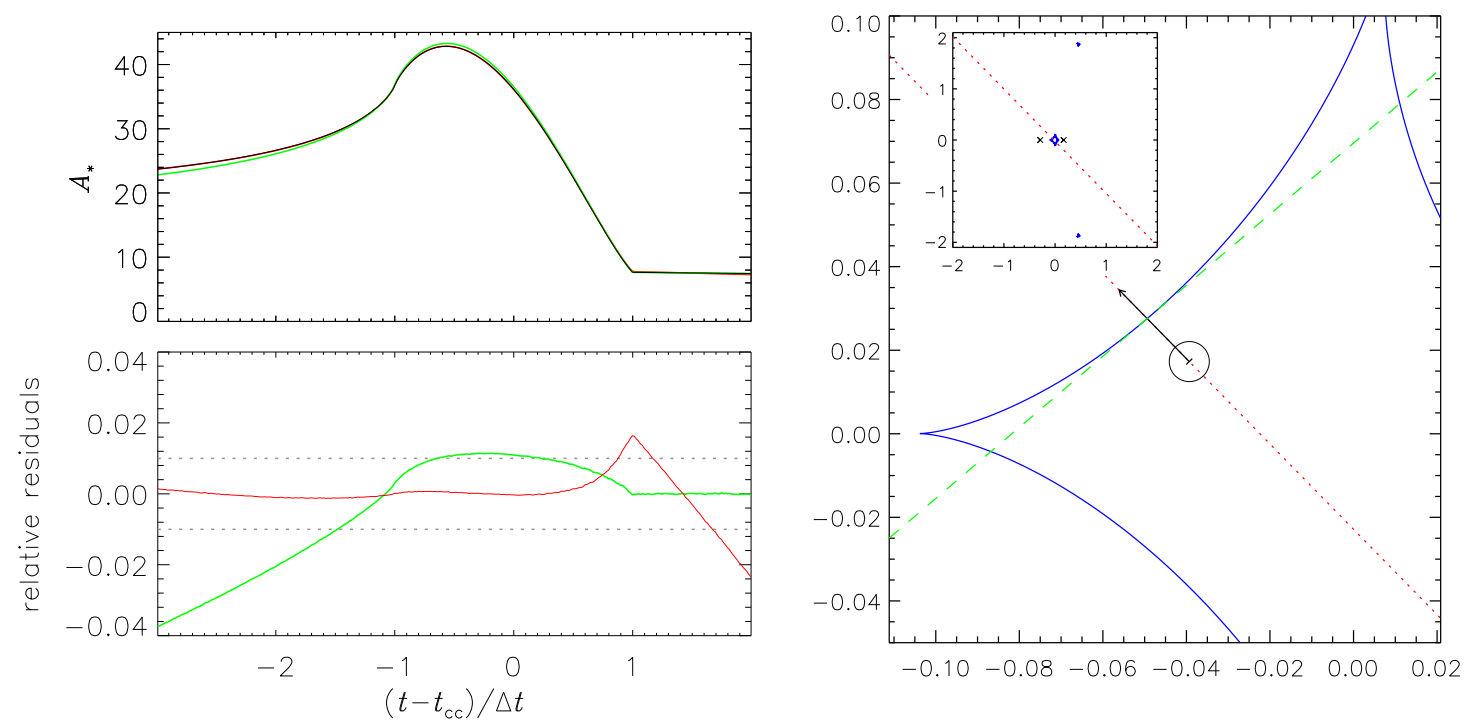

Figure 4: Validity of the linear fold approximation in OGLE-2002-BLG-069. Right panel shows the geometry of the second fold crossing in the close binary model of Kubas et al. (2005). Caustic is marked in blue, trajectory of the source in dotted red, tangent to the caustic at the point of crossing in dashed green and the part of the trajectory actually studied is marked by the black arrow. A wider view is presented in the inset. Top left panel compares the exact light curve (black) with the best-fit approximation of equation (4.1) fitting either all points simultaneously (red) or first the points where $G=0$ (green). Bottom left panel shows the corresponding residuals.

effect is reached when a source is positioned about one $\rho_{*}$ from the cusp on either side of the axis (where the same high-amplification regions give this time higher weight to the limb of the source). Just like in the case of sensitivity to an extended source, trajectories $A$ and $C$ that go perpendicular to the fold and axis of the cusp, respectively, scale linearly with source size. Trajectory B exhibits the same $\rho_{*}^{-1 / 3}$ scaling as was shown in section 2.2 and reaches $\delta_{\text {chr }}=0.01$ at $2.6 \rho_{*}$ outside the cusp for the larger source and at $6.0 \rho_{*}$ for the smaller source, respectively.

\section{Validity of linear fold approximation}

Light curves of some of the observed fold-crossing events were analyzed with the method of Albrow et al. (1999). Portions of the light curve sufficiently far from the caustic are modelled by a point source while for the remaining parts it is assumed that the source is crossing an idealized linear fold. The amplification of two of the extended-source images is obtained by cross-correlation of a source surface brightness profile with the inverse-square-root singularity of the fold. The amplification of the remaining three images is assumed to be a linear function of time $t$. The total amplification $A_{*}^{\text {appr }}$ reads

$$
A_{*}^{\mathrm{appr}}=U_{*} G\left(\frac{t-t_{\mathrm{cc}}}{\Delta t}\right)+A_{\mathrm{cc}}+\omega\left(t-t_{\mathrm{cc}}\right)
$$

where $G$ is a characteristic function vanishing outside the caustic, $t_{\mathrm{cc}}$ is the time of the fold crossing and $U_{*}, \Delta t, A_{\mathrm{cc}}$ and $\omega$ are other constants.

To check the applicability of this method we compute an exact light curve of one caustic crossing here and compare it with the best-fit light curve using equation (4.1). Based on the analysis 
of Kubas et al. (2005) we've selected OGLE-2002-BLG-069 as a favorable case for the method. In Figure 4 we plot the geometry of the situation, the approximate and exact light curves, and relative residuals $\left(A_{*}-A_{*}^{\text {appr }}\right) / A_{*}$. Obviously, even for a nearly perpendicular fold crossing relatively far from a cusp the residuals may reach well above $1 \%$ and should be seen in the data. Although most of the recent light-curve analyses don't use the approximation of equation (4.1) for the final parameter estimation, spectral changes during two-point-mass microlensing have been studied entirely using the linear fold approximation. This is true particularly for EROS-BLG-2000-5 (Albrow et al., 2001; Castro et al., 2001) where a nearly-parallel fold crossing occurred in the close vicinity of the cusp and the residuals of the linear fold approximation reach several tens of percent. Therefore, we advise against using the linear fold approximation without checking its validity for the studied caustic crossing..

\section{Discussion and conclusions}

We have investigated in detail extended-source effects in two-point-mass microlensing. While the largest differences between point-source and extended-source amplifications occur close to the caustics, we found significant sensitive areas between facing cusps of the caustic components where the relative excess can reach over $1 \%$. Chromaticity exhibits the same pattern away from the caustic but the details in the vicinity of the caustic differ. Based on analytical estimates we explain the nonlinear scaling of the contour extent along the outer axes of cusps. We found that the linear fold approximation can produce significant residuals even in favorable cases, and argue that its validity should be checked on every use.

\section{Acknowledgments}

This research was supported by Czech Science Foundation grant GAČR 205/07/0824. OP is thankful to the conference organizers for financial support.

\section{References}

Albrow, M. D., et al. 1999, ApJ, 522, 1022

Albrow, M. D., et al. 2001, ApJ, 550, L173

Castro, S., Pogge, R. W., Rich, R. M., DePoy, D. L., \& Gould, A. 2001, ApJ, 548, L197

Kubas, D., et al. 2005, A\&A, 435, 941

Mao, S., \& Paczyński, B. 1991, ApJ, 374, L37

Pejcha, O., \& Heyrovský, D. 2007, ApJ, submitted

Schneider, P., \& Weiß, A. 1992, A\&A, 260, 1

Vermaak, P. 2000, MNRAS, 319, 1011

Zakharov, A. F. 1995, A\&A, 293, 1

Zakharov, A. F. 1999, Astronomical and Astrophysical Transactions, 18, 17 